Draft version May 7, 2021

Preprint typeset using $\mathrm{IAT}_{\mathrm{E}} \mathrm{X}$ style emulateapj v. 5/2/11

\title{
CONTINUUM INTENSITY AND [O I] SPECTRAL LINE PROFILES IN SOLAR 3D PHOTOSPHERIC MODELS: THE EFFECT OF MAGNETIC FIELDS
}

\author{
D. FABBIAN \\ Instituto de Astrofísica de Canarias (IAC), E-38205 La Laguna, Tenerife, Spain; damian@iac.es \\ AND \\ F. Moreno-INSERTis ${ }^{1}$ \\ Instituto de Astrofísica de Canarias (IAC), E-38205 La Laguna, Tenerife, Spain; fmi@iac.es \\ Draft version May 7, 2021
}

\begin{abstract}
The importance of magnetic fields in three-dimensional magnetoconvection models of the Sun's photosphere is investigated in terms of their influence on the continuum intensity at different viewing inclination angles, and on the intensity profile of two [O I] spectral lines. We use the RH numerical radiative transfer code to perform a posteriori spectral synthesis on the same time-series of magnetoconvection models used in our publications on the effect of magnetic fields on abundance determination. We obtain a good match of the synthetic disc-centre continuum intensity to the absolute continuum values from the FTS observational spectrum; the match of the centre-to-limb variation (CLV) synthetic data to observations is also good, thanks, in part, to the 3D radiation transfer capabilities of the RH code. The different levels of magnetic flux in the numerical time-series do not modify the quality of the match. Concerning the targetted [O I] spectral lines, we find, instead, that magnetic fields lead to non-negligible changes in the synthetic spectrum, with larger average magnetic flux causing the line to become noticeably weaker. The photospheric oxygen abundance that one would derive if instead using non-magnetic numerical models would thus be lower by a few to several centidexes. The inclusion of magnetic fields is confirmed to be important for improving the current modelling of the Sun, here in particular in terms of spectral line formation and of deriving consistent chemical abundances. These results may shed further light on the still controversial issue regarding the precise value of the solar oxygen abundance.
\end{abstract}

Subject headings: magnetohydrodynamics (MHD) - radiative transfer — Sun: abundances — Sun: granulation - Sun: magnetic fields - Sun: photosphere

\section{INTRODUCTION}

The Sun is magnetised over its whole surface, even in the so called "quiet" regions (e. g., Truiillo Bueno et al. 2004; Solanki 2009; Sánchez Almeida \& Martínez González 2011: Bellot Rubio \& Orozco Suárez 2012; Nordlund et al. 2009), with at least as much magnetic flux present in the quiet Sun itself as in active regions (Gošić et al. 2014; Thornton \& Parnell 2011). The presence of ubiquitous magnetic fields has an influence on the average temperature stratification in the solar photosphere, thus indirectly affecting the many spectral lines formed there (see Fabbian et al. 2010, 2012, called Papers I and II, respectively, in the following). This, in turn, can have an impact on the accuracy of the chemical composition derived from different spectral features, when non-magnetic atmospheric models, even current generation ones accounting for departures from homogeneity (e.g., Stein \& Nordlund 1998; Asplund et al. 2000; Frevtag et al. 2002; Asplund 2005; Scott et al. 2014), are employed for solar abundance derivation purposes.

Three-dimensional (3D) convection simulations have already been extensively applied to aid in the understanding of different solar phenomena, including the im-

1 Also affiliated with: Departamento de Astrofísica, Universidad de La Laguna (ULL), E-38206 La Laguna, Tenerife, Spain pact of the convective velocity field on the formation of spectral lines in terms of their widths, shifts, shapes and asymmetries (e. g., Dravins et al. 1981; Dravins 1982; Nordlund et al. 2009). Generally, though, the effects of magnetic fields on the determination of solar chemical abundances has not been systematically explored. In Papers [I and III, we carried out a first quantitative assessment of the impact of magnetic fields in $3 \mathrm{D}$ convection models on the determination of the solar chemical composition. To that end, we calculated time-series of magnetoconvection simulations using the Copenhagen STAGGER radiation magnetohydrodynamics code. The quality of those simulations was confirmed in follow-up studies, where the snapshots were tested for thermodynamical and polarisation properties (Beck et al. 2013, 2015), as well as being employed for associating G-band bright points with magnetic field strengths of a few $\mathrm{kG}$ (Criscuoli \& Uitenbroek 2014), and for comparing numerically-predicted and observationally-derived velocity fields in solar convection via Fourier power spectra (Yelles Chaouche et al. 2014). Our 3D magnetohydrodynamical (MHD) models were also used for the interpretation of solar irradiance measurements, whereby the predictions of the simulations may provide a possible explanation for the counter-phase variation of the spectral irradiance at visible and IR wavelengths with the solar activity cycle (Criscuoli \& Uitenbroek 2014). 
The motivation for the present paper is to further test the quality of our magnetoconvection models and to proceed in the investigation of the effect of magnetic fields on the average spectra and derived chemical composition of the solar photosphere. Using the MHD simulations of Papers II and II,

(1) we attempt an absolute match of the synthetic continuum radiation to disc-centre FTS observations;

(2) we carry out a study of the continuum intensity variation when moving from disc centre to limb; and

(3) we investigate whether those models also correctly reproduce selected [O I] absorption lines in the solar spectrum.

Concerning item (1), Trujillo Bueno \& Shchukina (2009) used a single 3D HD snapshot by Asplund et al. (2000) to produce a synthetic disc-centre continuum intensity spectrum. Values at wavelengths in the visible part of the spectrum proved to be roughly $10 \%$ below observations. In Papers $\mathrm{I}$ and $\mathrm{II}$, using $1.5 \mathrm{D}$ radiation transfer calculations, we obtained a match of the absolute values of the synthetic continuum intensity at disc centre to within $\sim 7 \%$ of the solar observational data. In the present work, we see how this match can be substantially improved by using a different spectral synthesis code (RH, Uitenbroek 2001, 2002), with, therefore, opacities different to those used in our previous papers.

Concerning item (2), i. e. the continuum CLV behaviour, Koesterke et al. (2008) found that shortcomings in CLV results from the 3D HD model of Asplund et al. (2000) were roughly comparable to the shortcomings of classical 1D models. The results indicated the presence of too steep a temperature gradient in the 3D HD model. They tentatively concluded that the improved description of the convective energy transport in $3 \mathrm{D}$ may be offset by deficiencies introduced by a too simplified radiation transfer scheme. Including more opacity bins and additional sources of continuum opacities led Pereira et al. (2013) (called Paper III in the following) to achieve very good agreement of their CLV results with observations. It is thus of interest to check how well our 3D MHD models match the observations concerning the continuum CLV behaviour, in spite of the reduced number of opacity bins, by using the $3 \mathrm{D}$ radiation transfer capabilities of the $\mathrm{RH}$ code.

In the final part of the current work, we will look at the effect of the magnetic fields in convection models on the formation of selected [O I] spectral lines. Pereira et al. have recently argued in Paper III that claims for revised abundances based on 3D MHD modelling are premature. On the other hand, Socas-Navarro (2014) very recently found an extreme sensitivity of the derived solar oxygen abundance to possible systematics (retrieving values in the range $\left.\log \epsilon(\mathrm{O})_{\odot}=8.70-8.90 \mathrm{dex}\right)$. Typical uncertainties, mainly intrinsic to employing atmospheric models, would thus be substantially higher than what is usually claimed in the corresponding literature. It is clear that the solar oxygen chemical abundance derivation is particularly difficult, because the corresponding absorption lines present in the solar spectrum are few and problematic (see review by Asplund 2005).

In this work, we will show that, barred the remaining uncertainties (on, e. g., continuum opacity, treatment of radiative transfer and non-local thermodynamic equilib- rium effects) that complicate the treatment of spectrum formation, as well as those affecting the solar spectrum observations (see, e. g., Beck et al. 2011), the MHD approach provides synthetic spectra that match very well both the solar continuum intensity at different inclination angles, as well as the [O I] $557 \mathrm{~nm}$ and $630 \mathrm{~nm}$ absorption lines.

In the following section, we explain the simulation setup, we inform on the input data chosen for the spectral synthesis computations, and briefly present the method adopted in this study. In sections 3. 4] and 5, we present the analysis of the theoretical results of interest on disccentre absolute continuum intensity, on centre-to-limb variation of the continuum intensity, and on continuum intensity normalised spectral line profiles, respectively, and compare them to available observational data. The paper ends with a discussion and conclusions (Sec. 6] and 7).

\section{SIMULATION SETUP AND RESULTING MAGNETIC FIELD STRATIFICATION}

\subsection{Setup of the numerical (M)HD simulations}

The 3D model atmospheres adopted as input for the spectral synthesis are based on snapshots selected from the statistically stationary regime of the convection in our previous (M)HD simulations (Papers I and II), which used input solar abundances of carbon, oxygen and iron of, respectively, $\log \epsilon(\mathrm{C})_{\odot}=8.55, \log \epsilon(\mathrm{O})_{\odot}=8.77$, and $\log \epsilon(\mathrm{Fe})_{\odot}=7.50$. The snapshots we employ in this study belong to the different MHD series in those papers, which had a vertical magnetic field of uniform strength implanted at time $t=0$ of $56 \mathrm{G}, 112 \mathrm{G}$ and $224 \mathrm{G}$, respectively (labelled "50 G", "100 G" and " 200 G" in the following). Additionally, we will also use here the " $0 \mathrm{G}$ ", i.e., purely $H D$, series of those papers.

The atomic and molecular transition data we employ in the intensity spectrum calculations are from the Vienna Atomic Line Database (VALD) and from the National Institute of Standards and Technology Atomic Spectra Database (NIST ASD). VALD and its development have been described by Piskunov et al. (1995), Rvabchikova et al. (1999), and Kupka et al. (1999). In particular, we made use of the recent (February 2014) third release of $\mathrm{VALD}^{2}$, and of NIST $\mathrm{ASD}^{3}$ in its online version 5 (Kramida et al. 2012).

We employed version 2 of the RH code (Uitenbroek 2001, 2002) to perform the 3D radiation transfer calculations (we refer to them as "R3D" in the rest of this article) on the basis of the input 3D (M)HD snapshots. In the solar atmosphere, both Rayleigh scattering (off neutral hydrogen and helium) and Thomson scattering (off free electrons) may cause a departure from LTE conditions even in the absence of NLTE spectral lines. These scattering effects are negligible for the continuum at disc centre, but may become progressively more important towards the limb, where higher layers are being sampled. We thus include Rayleigh-Thomson (RT) scattering in the calculations of this paper, with a view to the centre-to-limb variation calculations of Sect. 4. Vertically, we cut the input model to 64 depth points covering a physical depth of -0.5 to $0.5 \mathrm{Mm}$ approximately. Hori-

\footnotetext{
2 http://vald.astro.univie.ac.at/ vald3/php/vald.php

3 http://physics.nist.gov/asd
} 
zontally, the full sampling of the original snapshots was maintained for the centre-to-limb variation (CLV) runs, with 252 grid points over $6 \mathrm{Mm}$ in each direction, while the sampling was reduced to 63 points in each horizontal direction over the same physical extent for the spectral line synthesis calculations.

We first computed the absolute intensity spectrum at disc-centre. Then, we performed the CLV runs using a Gauss-Legendre set with 9 inclination angles and $4 \mathrm{az}-$ imuth angles per octant (thus providing 36 angles per octant, or 144 rays in total). In sections 3 and 4 we will be comparing the $\mathrm{RH}$ results of this paper with those of simpler (e. g., $1.5 \mathrm{D}$, or without $\mathrm{RT}$ scattering) radiation transfer calculations carried out with other codes: the corresponding details will be explained in those sections. For the spectral line calculations, which focused on the $557 \mathrm{~nm}$ and the $630 \mathrm{~nm}$ oxygen features, the radiative transfer problem was solved using a fine $(10 \mathrm{~m} \AA)$ and uniform sampling in $\lambda$ space. The resulting output spectrum was set to cover $1.2 \AA$ around each of the two oxygen spectral lines of interest (which allowed us to account for any significant blends affecting them).

The spectral synthesis results for the MHD case were obtained neglecting the spectral line broadening due to the Zeeman effect, on the basis of the expectation that, compared to the indirect effect due to temperature changes, this direct effect of magnetic fields on spectral lines is relatively small. It in fact increases with $\lambda^{2}$ and it thus generally becomes appreciable in the infrared (IR), rather than in the visible wavelength range where the spectral lines we selected for this study fall.

\subsection{Average magnetic field distribution in the convection cells}

Some of the results presented in this paper are associated with the introduction of magnetic field in convection models, which can induce significant stratification changes, with consequent effects on spectral lines. Thus, for a better appreciation of our findings, it is adequate to consider briefly the average values of the magnetic field in the snapshots that serve as a basis for the radiation transfer calculations. The upper panel of Fig. 11 shows, using solid curves, the height distribution of the average magnetic field intensity, with the average being carried out separately for each horizontal plane and along time; the figure, therefore, shows the discrete version of:

$$
\langle B\rangle(z)=\frac{1}{t_{f}-t_{0}} \frac{1}{A} \int_{t_{0}}^{t_{f}} d t \int_{\substack{\text { horizontal } \\ \text { plane }}} d x d y B(x, y, z, t)
$$

with $A$ the area of the horizontal plane in the numerical box and $t_{0}$ and $t_{f}$ the limits of the time interval used for the averaging. Here, and for all subsequent figures in this subsection, the time averaging is done using the last 100 snapshots (corresponding to 50 solar minutes) of the statistically stationary regime in the simulations, with snapshots separated by 30 solar seconds. The different series are identified with blue, green and red colour for the "50 G", "100 G" and "200 G" series, respectively. All three $\langle B\rangle$ distributions decrease smoothly with height in the topmost Megameter of the convection zone and reach values of $\sim 80 \mathrm{G}, \sim 150 \mathrm{G}$, and $260 \mathrm{G}$, respectively, at $z=0$. In the highest layers (i.e., roughly in the topmost $150 \mathrm{~km}$ ), the distributions show an upturn, with the value of the mean magnetic field strength increasing to a maximum of $\sim 100 \mathrm{G}, \sim 170 \mathrm{G}$, and $\sim 300 \mathrm{G}$, respectively for the " $50 \mathrm{G}$ ", " $100 \mathrm{G}$ " and " $200 \mathrm{G}$ " series.

It is worth noting that the " $100 \mathrm{G}$ " $\langle B\rangle$-distribution of Fig. 11 is not far from the distribution shown by Shchukina \& Trujillo-Bueno (2011, 2015) to match the scattering polarization signal (induced by the Hanle effect) from a height of around $250-300 \mathrm{~km}$ (Gurtovenko \& Kostyk 1989), as derived from observations of the Sr I $460.7 \mathrm{~nm}$ line, simultaneously allowing for the explanation of the polarization signal (induced by the Zeeman effect) from a height of around $60 \mathrm{~km}$, as derived from observations of the Fe I $630.25 \mathrm{~nm}$ line. The small-scale local dynamo models of Vögler \& Schüssler (2007) or Rempel (2014), instead, lead to too weak average magnetic field strength values to explain the Sr I $460.7 \mathrm{~nm}$ Hanle-effect data.

The upper panel of Fig. 1 also shows (thick dashed curves) the height dependence of the root mean square (rms) magnetic field intensity, $B_{r m s}=\sqrt{\left\langle B^{2}\right\rangle}$, which also serves to illustrate the distribution of magnetic energy in the box in the stationary regime. The $B_{r m s}$ curves peak (with values of $\sim 300 \mathrm{G}, \sim 460 \mathrm{G}$, and $\sim 670 \mathrm{G}$, respectively) in progressively higher layers (between $\sim 1700$ and $\sim 1200 \mathrm{~km}$ ) as magnetic flux increases between the " $50 \mathrm{G}$ " and the " $200 \mathrm{G}$ " case, then decrease monotonically.

The dotted horizontal lines in the figure mark the constant value of the vertical component of the field that was implanted at $t=0$ in each series. In all cases, in the stationary regime the $B_{r m s}$ field is larger than the average field intensity. This is a result of the intermittency created in the field by the action of the convective flows. To show this effect from a different perspective, we plot in the lower panel of Fig. 1 the ratio $\sqrt{\left\langle B^{2}\right\rangle} /\langle B\rangle$. This ratio can serve as an index for the intermittency level in each of the time-series. The ratio is seen to increase monotonically with height in the subsurface layers, and to reach a maximum very near the surface, $z=0$. This is expected since the Alfven Mach number of the convection flows is largest near the surface. We also see that the intermittency level decreases the larger the initially implanted field, with peak ratios of $\sim 3.0,2.4$ and 2.1 for the "50 G", "100 G" and "200 G" series, respectively. Above the surface, the intermittency ratio decreases sharply, given the less passive role of the magnetic field generally for higher atmospheric levels and the decreasing intensity of the convection there.

\section{DISC-CENTRE ABSOLUTE CONTINUUM INTENSITY}

In this section we present the first group of results in the paper, namely the comparison with observations of the absolute disc-centre continuum intensity values obtained through spectral synthesis calculations from our (M)HD snapshots (Fig. 2, upper panel). For the observations we took the Fourier Transform Spectrograph (FTS) data, drawn in the figure as a collection of points in light gray. For the comparison, a pseudocontinuum is needed, to use as an envelope to the FTS data. We obtained it (black line) by linking the local maxima of the original FTS data, bridging gaps across wavelength regions 

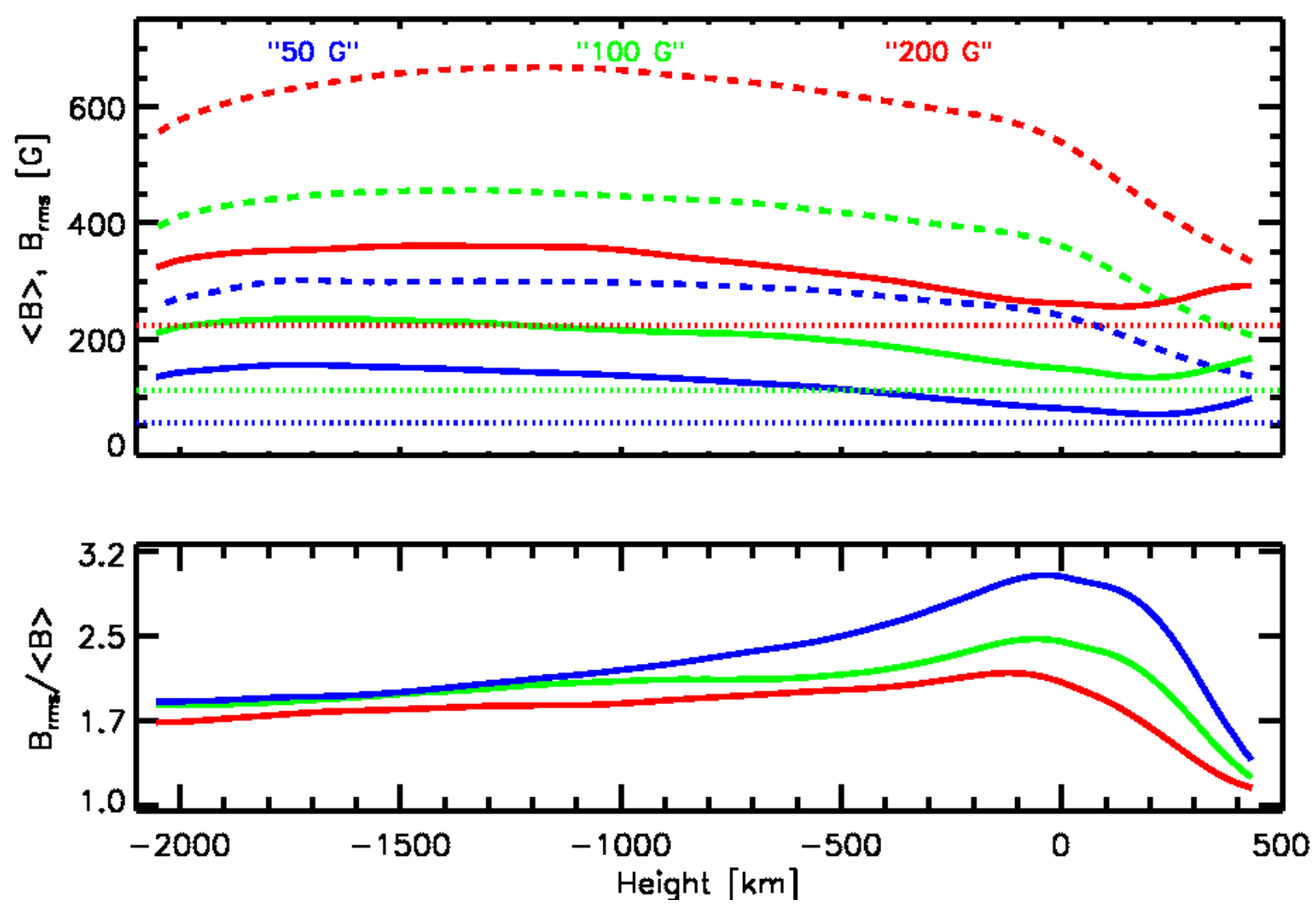

Figure 1. Upper panel: height-stratification of the average magnetic field intensity (solid curve) and of the root-mean-square magnetic field (thick dashed curve), expressed in Gauss units. Blue colour is used for the "50 G" series, green for the " 100 G" series, and red for the " 200 G" series. The dotted horizontal lines mark the constant value of the horizontally-averaged vertical component of the signed magnetic field intensity for the three series. Lower panel: height-stratification of the ratio of the rms magnetic field intensity, $B_{r m s}$, and of the average magnetic field intensity $\langle B\rangle$. Same colour coding as for the upper panel.

affected by strong absorption features. Our R3D data are shown as blue diamonds and they represent the absolute continuum intensity values obtained using the threedimensional radiation transfer calculation explained in Subsection 2.1. To take into account the fluctuations in time, we calculated the value of the continuum at each of the wavelengths as the time average of 21 snapshots covering 50 solar minutes, with the first snapshot taken at 100 solar minutes inside the statistically stationary part of the simulation. The theoretical values (the blue diamonds) are very near the FTS continuum, as also apparent in the lower panel of the figure: the average mutual deviation is $\sim 3.8 \%$, with the minimum and maximum relative difference being $\sim 1.5 \%$ (at $600 \mathrm{~nm}$ ) and $\sim 6 \%$ (at $900 \mathrm{~nm}$ ), respectively.

In the upper panel of Fig. 2, we also show, as red triangles, our previous results of Paper II, obtained with a different radiation transfer calculation, namely, using the Lilia code of Socas-Navarro (2001) and without any RT scattering. The better match to observations of our current results compared with those of Paper II provides reassurance both on the quality of our models and on the opacities used by the RH code. We further tested the effect of not including Rayleigh and Thomson scattering in our current $\mathrm{RH}$ calculations. We found the change in absolute continuum intensity to be unimportant.

The small remaining percentage difference (shown in Fig. 2, lower panel) of the R3D results with respect to FTS can be understood as partly due to the fact that the (M)HD snapshots have a slightly lower (within $\lesssim 20 \mathrm{~K}$ ) outgoing radiation flux compared to the measured solar one. However, that can explain only $\lesssim 1 \%$ of the discrepancy. A more significant contribution to the $\sim 3.8 \%$ average difference with FTS may arise from the comparatively low number of opacity bins used in our Stagger-code calculation. A thorough treatment of line blocking/blanketing effects by using very accurate atomic data for many spectral lines may also provide a further improvement of the match with observations, an approach which can, however, become prohibitively timeconsuming when coupled with $3 \mathrm{D}$ radiation transfer, due to the plethora of lines present in the solar spectrum that need to be taken into account. In any case, the FTS atlas, like any observation, is, itself, affected by uncertainties of up to a few percent (for example, affecting regions of strong telluric absorption, see Burlov-Vasilijev et al. (1998)), which contribute to part of the difference seen here.

The comparison between the theoretical results for HD and MHD runs is also of interest. It turns out that there is only a small difference between them; in fact, the HD curve would be nearly indistinguishable if plotted alongside the blue diamonds of Fig. 2 (which correspond to the R3D MHD results for the "200 G" case): the HD continuum is only $\Delta(I) \lesssim 0.02 \cdot 10^{4} \mathrm{~W} \mathrm{~m}^{-2}$ sr nm below the MHD one. The reason why their continuum level turns 


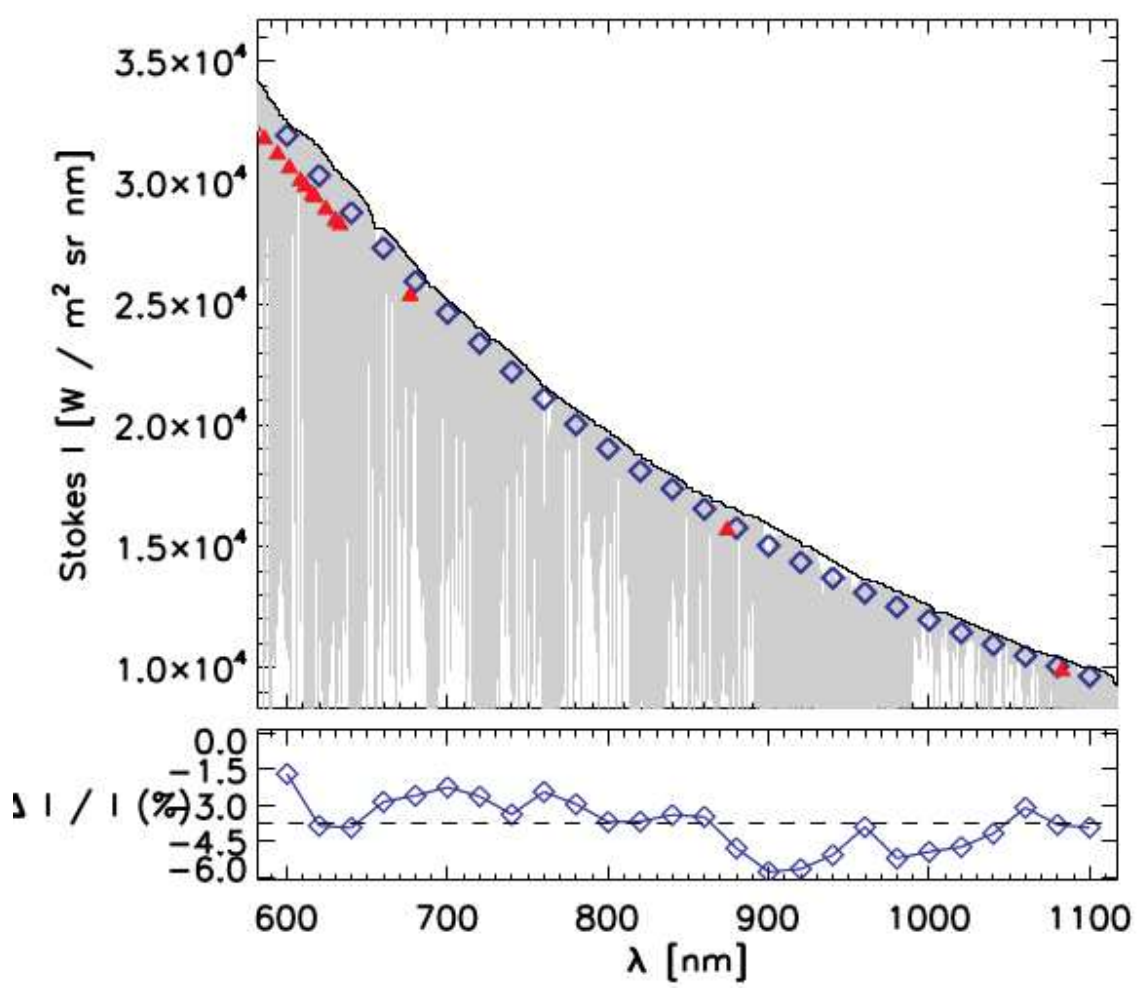

Figure 2. Absolute disc-centre continuum intensity at different wavelengths (upper panel). The solar atlas data from FTS observations are shown in light grey colour. The theoretical R3D values are plotted as blue diamond symbols. Our FTS pseudocontinuum is shown as a solid black curve. Our R1.5D values of Paper II are shown as red triangles. In the lower panel, we show the percentage difference between the R3D results and the FTS pseudocontinuum. The dashed horizontal line marks the $\sim-3.8 \%$ average (R3D MHD - FTS) deviation level. 
out to be nearly identical is that (Paper II, Fig. 3; Paper II, Fig. 1) the MHD-HD temperature difference tends to be smallest (tending to negligible) precisely in the layers where the continuum forms.

\section{CENTRE-TO-LIMB VARIATION OF THE CONTINUUM INTENSITY}

As an observer's line-of-sight moves towards the solar limb, the continuum intensity decreases because it originates in higher layers which are not as bright (warm) as the deeper ones to which the observer's line-of-sight penetrates when looking at disc centre (e.g., Grav 1992, chapter 9). We can thus use the behaviour of the theoretical CLV to verify whether the temperature stratification of the models results in a proper matching of the solar continuum intensity CLV.

In Fig. 3, we show our results on continuum intensity centre-to-limb variation (CLV) for the synthetic spectra normalised to the corresponding value at disc centre. We studied the continuum intensity behaviour both against $\mu_{\mathrm{z}}=\cos \theta_{\text {helio }}$ (upper panel), with $\theta_{\text {helio }}$ the heliocentric angle, as well as against wavelength (lower panel) in the range $600 \mathrm{~nm}$ to $2400 \mathrm{~nm}$. In the upper panel of Fig. 3. the theoretical R3D continuum intensity results from our computations for the MHD case are represented as diamond symbols linked by dashed segments, while observations are shown as '+' signs for Neckel \& Labs (1994) and ' $x$ ' symbols, for Pierce et al. (1977). Our R3D results are seen to match very well the solar limb darkening observational data. Taking into account radiation transfer effects properly in a 3D inhomogeneous atmosphere thus produces realistic values of the average radiation in the continuum. In the figure we are also showing (as triangles linked by a dotted line) the results obtained using a simplified radiation transfer calculation with the Nicole code of Socas-Navarro et al. (2014) (which, like the Lilia code before, uses a different opacity package than $\mathrm{RH}$ ). The Nicole version used had a 1.5D approach to the horizontal radiation transfer (called "R1.5D" in the following). In that approach, we considered as many $\mu$ angles as in the full 3D calculation just shown, but the horizontal radiation transfer coming out of a given pixel is calculated via an infinite repetition of the corresponding column in the two horizontal directions. The R1.5D results are found to deviate substantially from the observational data: differently from the previous Section's result on disc-centre continuum intensity, the continuum CLV values are not only sensitive to adopted opacities, but are also sensitive to horizontal transfer effects, especially as one moves toward the limb. It is then natural that the $\mathrm{R} 1.5 \mathrm{D}$ results deviate importantly from the observations when approaching the limb, since they do not take proper account of the hills and valleys of the $\tau$ isosurfaces in the 3D models.

The lower panel of Fig. 3 compares the observational data and theoretical results just discussed, now against wavelength. This more clearly highlights the differences

\footnotetext{
4 Note that the Neckel \& Labs (1994) data agree well with the less recent ones by Pierce \& Slaughter (1977) (not shown here) in the wavelength interval of $303-730 \mathrm{~nm}$ common to the two datasets. In the adjacent common wavelength interval of $740-$ $1100 \mathrm{~nm}$, the Neckel \& Labs (1994) data agree well with those of Pierce et al. (1977). For this reason, we show here the values for the latter dataset only for the non-overlapping wavelength region above $1100 \mathrm{~nm}$.
}

between the R3D and R1.5D approaches. The symbols are the same as in the upper panel of this figure (diamonds for R3D, triangles for R1.5D, ' + ' signs and ' $x$ ' for the observations), except without colour coding in wavelength. Again here, the R3D-R1.5D deviations (i. e., those due to horizontal transfer effects) generally increase when moving toward the limb, in particular, at longer wavelengths. This is in line with the results in Paper III (their Fig. 3), where a similar behaviour is visible in the case of the classical MARCS and PHOENIX 1D solar photosphere models (while the 1D Holweger \& Müller model, being semiempirical, not surprisingly matches the observational data as well as their 3D models). It should be noted that the comparison of theoretical and observational data very close to the limb (i.e., at $\mu_{z}<0.2$ or, equivalently, $\theta_{\text {helio }} \gtrsim 80$ degrees) is less helpful, since the latter data tend to become less reliable there.

In both panels of Fig. 3, we show only the "200 G" MHD case, for both the R1.5D and R3D radiative transfer calculations. The R3D case for the other series, in fact, generally displays a very similar match to observations as the one plotted. The situation is thus similar to what was already seen in the previous Section regarding the absolute continuum intensity, with relatively small differences between the results for the HD case and the MHD case(s).

The fine match to observations of our current R3D results again provides reassurance on the accuracy of our 3D (M)HD models and on the RH opacities and spectral synthesis. Differently from Sec. 3. though, it is harder to disentagle for these CLV runs how much of the difference between $\mathrm{R} 3 \mathrm{D}$ and $\mathrm{R} 1.5 \mathrm{D}$ results is due to, respectively, different opacities, Rayleigh and Thomson scattering (which should play more of a role at inclined angles), and pure geometrical / horizontal transfer (3D vs. $1.5 \mathrm{D})$ effects in the treatment of radiation.

\section{5. [O I] SPECTRAL LINE PROFILES}

In this section, we analyse the behaviour of the profile of selected spectral absorption lines when using 3D snapshots from our HD and MHD series and when changing the $\mathrm{C}$ and $\mathrm{O}$ solar abundance adopted as input to the spectral synthesis code. The main aim is to understand how the change in temperature stratification due to the presence of magnetic fields affects the spectral lines and to additionally verify whether our 3D MHD photospheric models coupled with $3 \mathrm{D}$ radiative transfer for the a posteriori spectral synthesis can fit observations better, or at least as well as when using input photospheric models from our HD series. For this part of our study, we selected two representative spectral lines of neutral atomic oxygen, namely [O I] 557 and [O I] $630 \mathrm{~nm}$, both produced by radiatively forbidden transitions. These two spectral lines are deep-forming and very weak in the solar spectrum. According to Gurtovenko \& Kostyk (1989), the main contribution to the formation of their core is from a range of layers around a height of, respectively, $\sim 130 \mathrm{~km}$ and $\sim 180 \mathrm{~km}$, above $\tau_{500 \mathrm{~nm}}=1$. Being formed in LTE, and given the scarcity of available oxygen lines in the solar spectrum, these spectral lines represent important tools in terms of the solar oxygen abundance determination (Meléndez \& Asplund 2008; Scott et al. 2009). Their use for the purpose of estimating the solar oxygen abundance is, however, sig- 

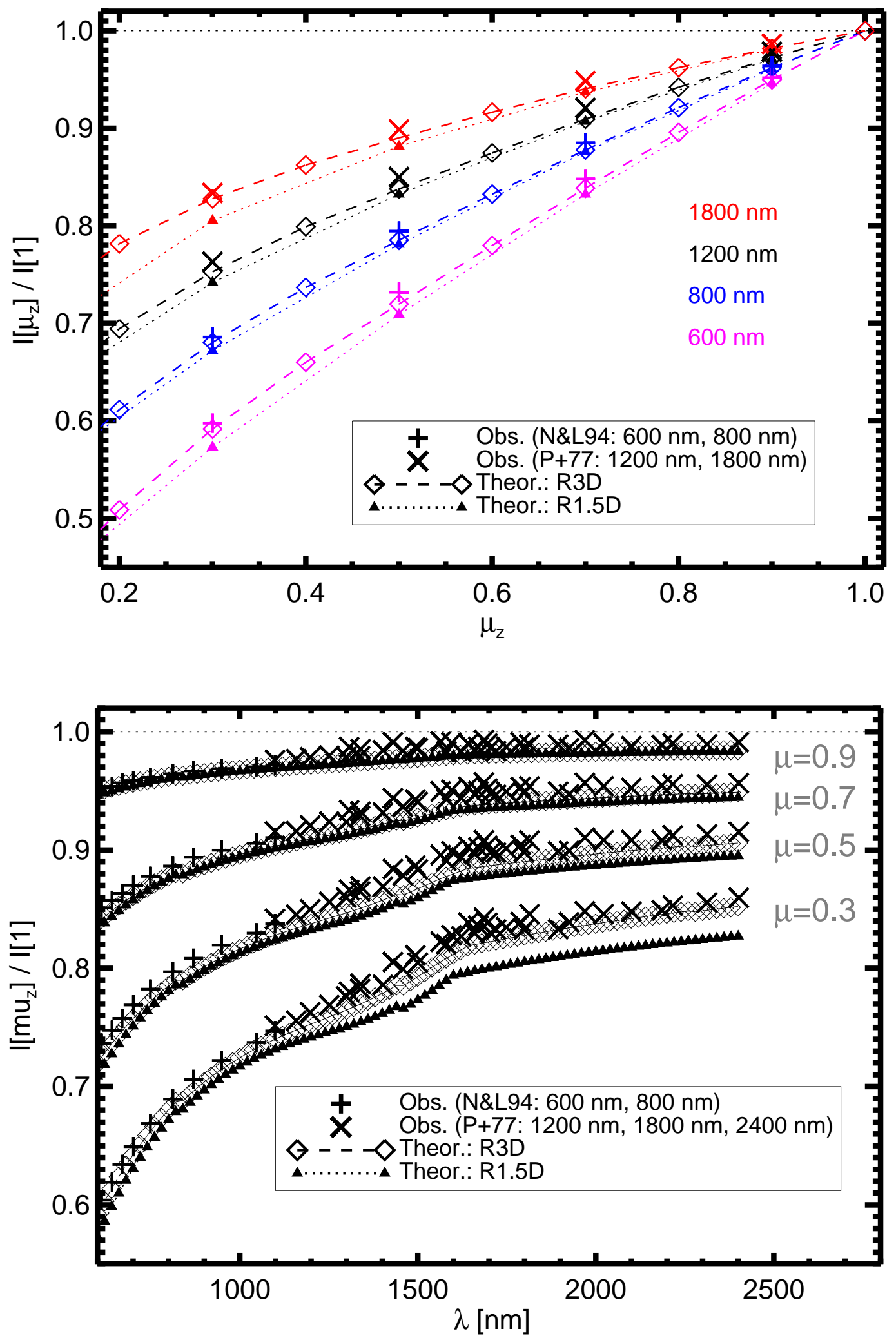

Figure 3. Upper panel: predicted continuum intensity at different wavelengths (indicated by the different colours, as labelled) from our R3D (this paper, diamond symbols linked by dashed segments) and R1.5D (Paper III, triangular symbols) computations for different values of $\mu_{\mathrm{z}}=\cos \theta$ (where $\theta$ is the heliocentric angle) is compared to solar limb-darkening observational data by (Neckel \& Labs (1994) (' + ' symbols), and by Pierce et al. (1977) (' $x$ ' signs). The dotted horizontal line indicates the normalisation level with disc-centre continuum intensity. Lower panel: the same, now versus wavelength and with the different curves being for different $\mu_{\mathrm{z}}=\cos \theta$ values, as labelled. Symbols are the same as for the upper panel, as marked in the legend. 


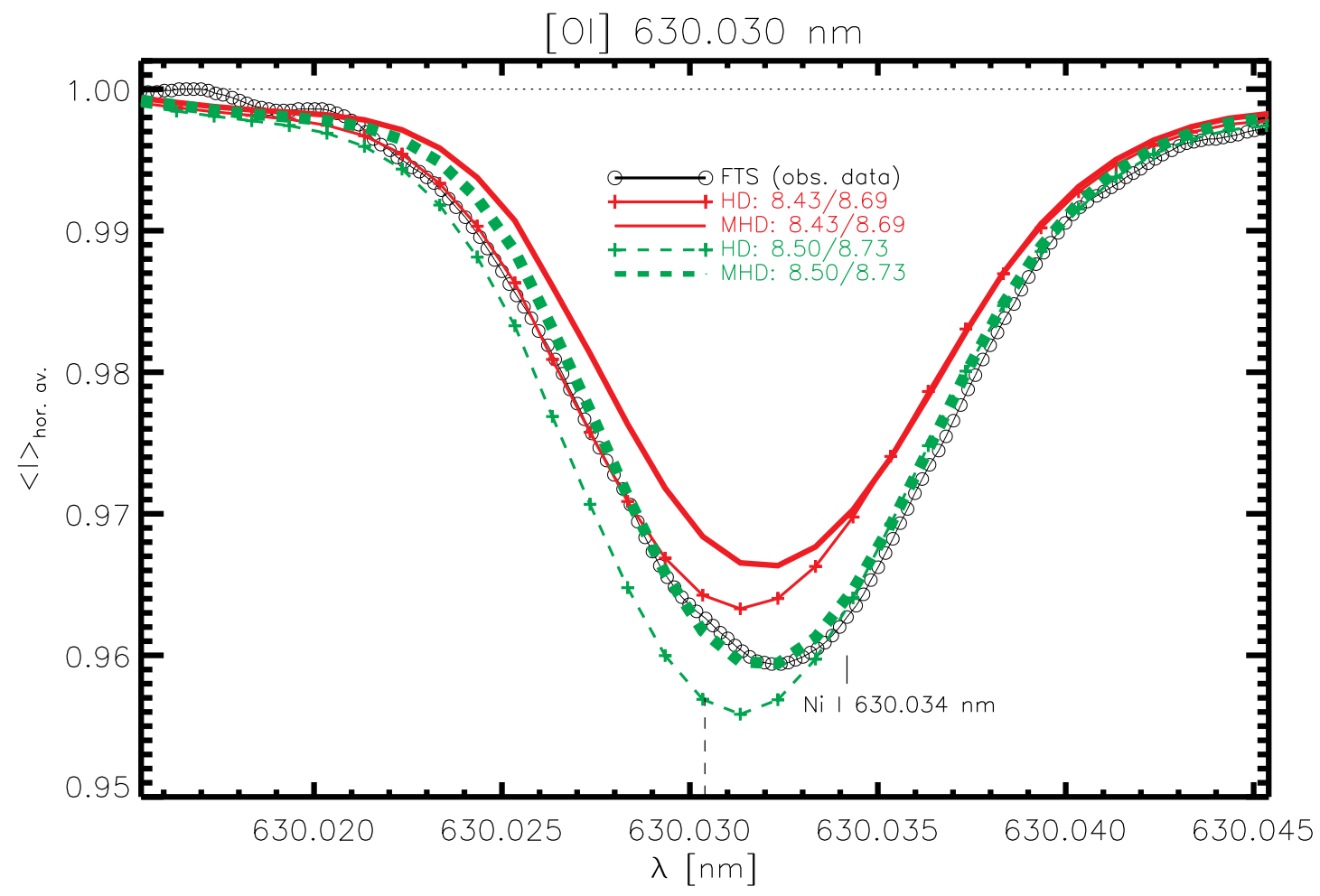

Figure 4. Solar observational disc-centre intensity data from FTS (black open circles) and R3D synthetic intensity profiles (coloured curves) in HD and MHD for the blended feature at $630 \mathrm{~nm}$, which contains the [O I] line of interest. As per the legend in the figure, the HD and MHD synthetic profiles shown are the result of adopting different choices for the input $\mathrm{C}$ and $\mathrm{O}$ solar abundances. The wavelength of the [O I] line and of the blending $\mathrm{Ni}_{\mathrm{I}}$ line are marked with a dashed vertical segment and with a solid vertical segment, respectively. The dotted horizontal line indicates the normalisation level with the adjacent continuum intensity.

nificantly affected by blends, mainly $\mathrm{C}_{2}$ molecular lines for the [O I] $557 \mathrm{~nm}$ absorption line, and a $\mathrm{Ni}$ I atomic line for [O I] $630 \mathrm{~nm}$. Additionally, the blending Ni I line requires proper treatment of its isotopic splitting, accounting for which significantly improves the fit to observational data. In the following analysis, we include all significant blends, among them the $\mathrm{C}_{2}$ and $\mathrm{Ni}$ I lines just mentioned. Note that, while free of significant known blends, the strong, widely used neutral oxygen triplet lines at $777 \mathrm{~nm}$ are prone to significant non-LTE effects (see Fabbian et al. 2009; Pereira et al. 2009) and thus would require a more complicated and time-consuming line synthesis treatment than the one we adopt here.

The [O I] 557 and [O I] $630 \mathrm{~nm}$ lines fall in the wavelength region where the absolute continuum intensity from our theoretical R3D calculations best matches the FTS solar atlas (see Fig. 2). We here compare the continuum-normalised theoretical spectra with Fourier Transform Spectrograph (FTS) observations of the solar spectrum. We normalised the spectral intensity across each of the wavelength regions to the relevant intensity of the adjacent pseudocontinuum. Note that for spectral lines around $600 \mathrm{~nm}$, the variation of the continuum level with wavelength across the line is negligible $\left(<10^{-4}\right)$ and does not need to be taken into account for our purposes.

The line formation calculations are computationally demanding, since many wavelength points must be used (as explained in Sec. 2.1) for an accurate study of the line profiles. Therefore, we selected as input model photosphere a single snapshot per series, namely one from the series with no magnetic field and another one from the "200 G" MHD series, respectively labelled "HD" and "MHD" in the following. Both snapshots were taken at a point in the calculations which corresponds to $100 \mathrm{~min}$ utes of solar time evolution in the statistically stationary regime of the convection. The choice to study the relevant effects based on the " 200 G" MHD series was made to estimate the expected maximum changes that the presence of magnetic fields induces - via a modified atmospheric temperature structure - on the profile of the selected oxygen spectral lines. Given the weakness of the latter, this also makes the representation of results in the figures of this Section clearer. This study thus covers quiet Sun to weak plage conditions.

In Figs. 4 and 5] we show the solar disc-centre intensity results of the R3D line synthesis for, respectively, the spectral region around the [O I] $630 \mathrm{~nm}$ and $557 \mathrm{~nm}$ absorption features. Our initial approach was to attempt to fit the FTS observations with the R3D synthetic spectra obtained using input abundance values from Asplund et al. (2009), in particular, $\log \epsilon(\mathrm{C})_{\odot}=8.43 \mathrm{dex}$ and $\log \epsilon(\mathrm{O})_{\odot}=8.69$ dex. The solar carbon abundance adopted is important here, since it affects the right calculation of the $\mathrm{C}_{2}$ concentration, which in turn is relevant due to the corresponding molecular blends affecting the [O I] $557 \mathrm{~nm}$ spectral feature. The formation of CO, instead, takes place at cooler temperatures than relevant here and is therefore unimportant for our results.

By comparing the corresponding HD and MHD synthetic intensity profiles (red curves in Figs. 4 and 5, re- 


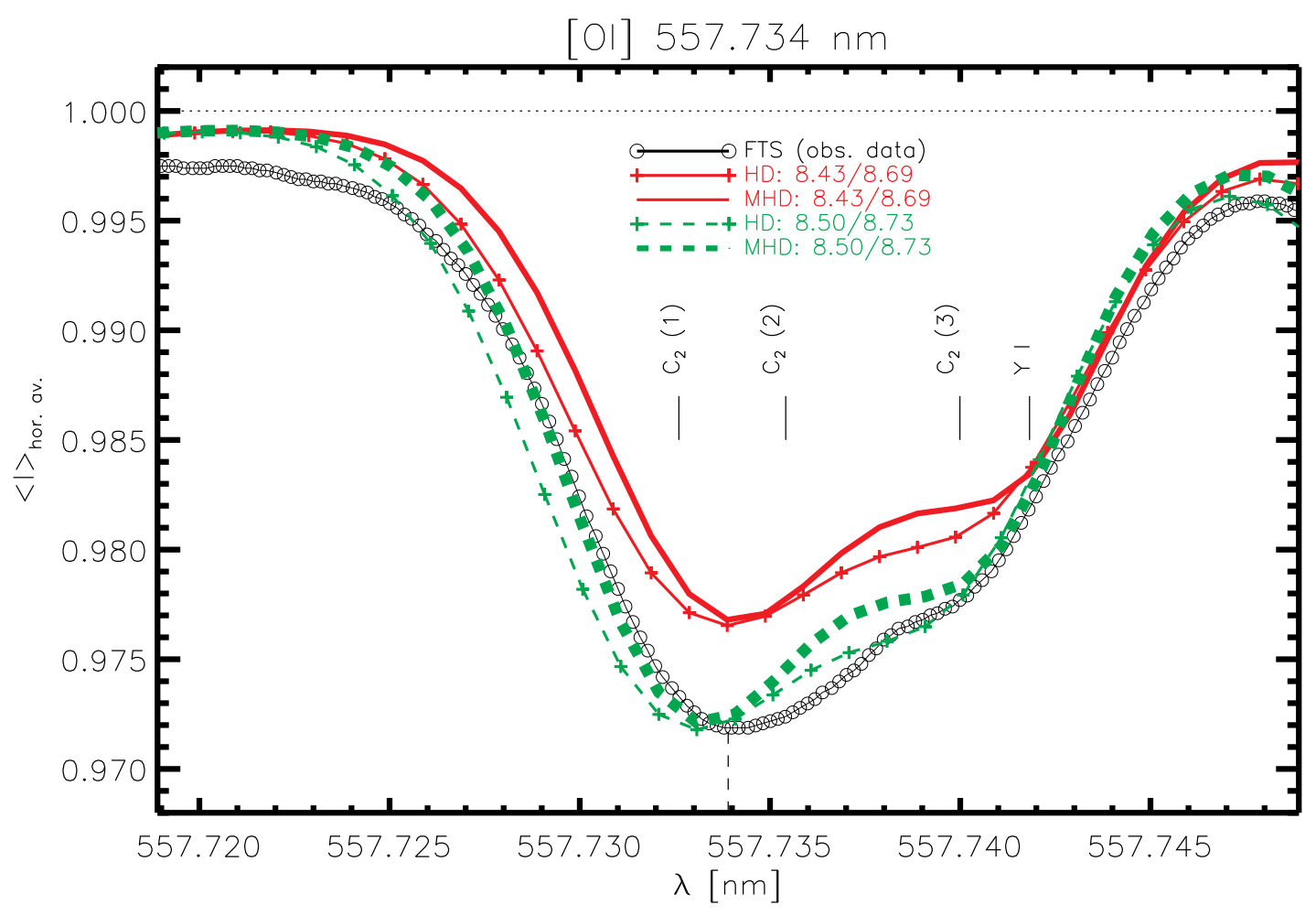

Figure 5. Solar observational disc-centre intensity data from FTS (black open circles) and R3D synthetic intensity profiles (coloured curves) in HD and MHD for the blended feature at $557 \mathrm{~nm}$, which contains the [O I] line of interest. As per the legend in the figure, the HD and MHD synthetic profiles shown are the result of adopting different choices for the input $\mathrm{C}$ and $\mathrm{O}$ solar abundances. The wavelengths of the [ $\left.\mathrm{O}_{\mathrm{I}}\right]$ line is marked with a dashed vertical segment, and the wavelengths of important blending spectral lines are marked with solid vertical segments $\left(\mathrm{C}_{2}(1)=\mathrm{C}_{2} 557.733 \mathrm{~nm} ; \mathrm{C}_{2}(2)=\mathrm{C}_{2} 557.735 \mathrm{~nm} ; \mathrm{C}_{2}(3)=\mathrm{C}_{2} 557.740 \mathrm{~nm} ; \mathrm{Y}_{\mathrm{I}}=\mathrm{Y}_{\mathrm{I}} 557.742 \mathrm{~nm}\right)$. The dotted horizontal line indicates the normalisation level with the adjacent continuum intensity.

spectively for [O I] $630 \mathrm{~nm}$, and [O I] $557 \mathrm{~nm}$ ), one clearly sees that both of the spectral absorption features we targetted become weaker in MHD, as expected due to the higher average temperature in the relevant line-forming layers. On the other hand, for both spectral lines, the fit against observations is not satisfactory when using those abundances. The lines are significantly too weak, in both MHD and HD. Not including blends would of course produce spectral profiles having the wrong shape because they would miss the additional asymmetry.

The poor fit of the red curves in Figs. 4 and 5 is to be expected because our (M)HD models have a different temperature stratification from the ones employed by Asplund et al. (2009). One may thus change the value of the elemental abundances adopted as input in the spectral synthesis, to verify the sensitivity of spectral features to a different chemical composition. We tested adopting different $\mathrm{C}$ and $\mathrm{O}$ input solar abundances and visually obtained a best fit when choosing the values $\log \epsilon(\mathrm{C})_{\odot}=8.50 \mathrm{dex}$ and $\log \epsilon(\mathrm{O})_{\odot}=8.73 \mathrm{dex}$. The green dashed curves in Fig. 4 and Fig. 5 show the profile of the targetted spectral lines when adopting these as input abundance values. The thick dashed curve represents the MHD case, while the thin curve with crosses is for the HD case. As seen in the figures, for both of the wavelength regions studied here, our synthetic lines for the MHD case match very well the disc-centre intensity profiles observed from the Sun, with the fit being particularly good in the case of the $630 \mathrm{~nm}$ spectral ab- sorption feature. It is noteworthy that this good agreement in both wavelength regions is achieved while using the same set of $\mathrm{C}$ and $\mathrm{O}$ solar abundance values, as one should require for consistency. Note that the abundance of $\mathrm{Ni}$ was maintained fixed at the value of Asplund et al. $(2009), \log \epsilon(\mathrm{Ni})_{\odot}=6.22 \operatorname{dex}$.

In the case of the $630 \mathrm{~nm}$ absorption feature, a crucial contribution to the composite intensity profile comes from accounting for the isotopic splitting of a blending neutral nickel feature present on its longer-wavelength side. We achieved this by scaling the Johansson et al. (2003) measured $\log g f$ for the nickel spectral line under consideration by the abundance of each of five Ni I isotopes (the same treatment adopted by Pereira et al. 2009, see their Sect. 4.2.2 and their Table 4). Regarding the $557 \mathrm{~nm}$ absorption feature, the presence of a small residual discrepancy - with respect to the FTS data - on the red side (at $\sim 557.738 \mathrm{~nm}$ ) of our R3D line profile obtained using our final adopted abundances, and seen in Fig. 5, may indicate that a weak, yet unknown blend might be present at that wavelength, just redward of the [O I] line. We note that Meléndez \& Asplund (2008) obtained a very good fit of the $557 \mathrm{~nm}$ wavelength region, at least in the case of the Delbouille solar atlas, when using their own spectrum synthesis code on a threedimensional hydrodynamical model of the solar atmosphere obtained by temporally and spatially averaging the snapshots from the series of Asplund et al. (2004). They derived values of $\log \epsilon_{\mathrm{C}} \sim 8.43$ based on fitting 
the blending $\mathrm{C}_{2}$ lines in the relevant wavelength region. With that value, they then found $\log \epsilon_{\mathrm{O}} \sim 8.70$ from the $557 \mathrm{~nm}$ absorption feature. Those abundance values are significantly $(\sim 0.03-0.07 \mathrm{dex})$ lower than our final adopted input $\mathrm{C}$ and $\mathrm{O}$ solar abundances.

\section{DISCUSSION}

In this study, we have obtained a good match of quantities resulting from our 3D (M)HD simulations against observational data. The studied quantities were: the $a b$ solute continuum intensity at disc centre, the CLV of the continuum intensity, and the profiles for two selected absorption features, each containing an oyxgen spectral line ([O I] $557 \mathrm{~nm}$ and $630 \mathrm{~nm}$, respectively) blended with lines of other chemical elements.

The continuum intensity (disc-centre and/or CLV) proves to be sensitive to adopted opacities and 3D radiation transfer capabilities of the spectral synthesis procedure, and corresponding observations at inclinations from disc centre to close to the limb are very well fit by our 3D models (Secs. 3 and 4). The good matching of absolute continuum intensity at disc centre (Sec. 3) confirms that the effective temperature of the deep-lying continuum-forming layers is realistic, but does not provide tight constraints for higher layers. The CLV of continuum intensity is a better tool for that purpose, and our results provide a quite satisfactory match (Fig 3) of solar limb darkening data, with the implication that the theoretical average temperature stratification should be relatively close to the correct one. The synthetic CLV values tend to be lower than the observations, in particular at intermediate angles $\left(\mu_{\mathrm{z}} \sim 0.5\right)$, when considering intensities normalized to the values at disc centre, as is customary in CLV studies. So, this deviation adds up to the error in the absolute matching found in Sec. 3 (average of $4 \%$, see Fig. 2). For an even more accurate fit of solar continuum data, lower opacities and/or increased radiative flux at $\sim 1400-1800 \mathrm{~nm}$ (possibly due to using a larger number of opacity bins in the simulations, or to atomic data improvements at those wavelengths), a mildly warmer temperature structure especially in intermediate to deep layers of the atmospheric model (thus, with a steeper temperature gradient at $\log \tau_{500} \mathrm{~nm} \gtrsim-1$ ) and/or more magnetic flux, may be required. Note that precisely in the wavelength range in question the line-ofsight penetration in the Sun reaches its maximum (Gray 1992, chapter 9), which would indicate the need for better modelling in those deep, warm layers. Alternatively, the larger discrepancy present especially around $1500 \mathrm{~nm}$ (see Fig. 3, lower panel) may imply that improved observations are required in that wavelength region.

Regarding the $\left[\begin{array}{ll}\mathrm{O} & \mathrm{I}\end{array}\right]$ spectral lines targetted in this study, we obtain a good match of the synthetic data with the corresponding observations. In particular, when employing the 3D MHD "200 G" photospheric model, the match is achieved for $\log \epsilon_{\mathrm{MHD}}(\mathrm{C})_{\odot}=8.50 \mathrm{dex}$ and $\log \epsilon_{\mathrm{MHD}}(\mathrm{O})_{\odot}=8.73$ dex, while neither the HD nor the MHD models are able to fit observations if adopting the lower solar abundances of Asplund et al. (2009), $\log \epsilon(\mathrm{C})_{\odot}=8.43$ dex and $\log \epsilon(\mathrm{O})_{\odot}=8.69 \mathrm{dex}$. To appreciate the effect of the magnetic fields with the largest possible clarity, in Sec. 5 we only showed results for the extreme cases of zero magnetic field and of the "200 G"
MHD time-series, the latter being the case with the highest vertical magnetic flux among our ensemble of simulations. Comparing these two cases, there is a clear effect of the modified average atmospheric temperature stratification on the line profile. However, the other MHD series ("50 G" and "100 G") are more likely to be representative of standard quiet Sun conditions. Additionally, for maximum accuracy when aiming at absolute solar chemical composition determinations, line broadening by the Zeeman effect should be included. Consequently, the final solar carbon and oxygen abundances that we could deduce from the present study may be somewhat different than obtained in Sec. 5] This needs to be checked in detail using a larger number of spectral lines - and, ideally, even better observational data - for the chemical elements in question, in future studies. In any case, it is a significant result that, due to the sensitivity of the profiles to the temperature stratification, differential effects are present for the two oxygen lines studied, and that with an appropriate choice of input chemical abundances, our MHD models can provide a good match of the corresponding observed spectra. Magnetic effects are therefore non-negligible, and given the observational evidence of the presence of magnetic fields all over the Sun, they should be taken into consideration. One may achieve a match of the HD model to the observed line spectra by raising the abundances a little from the level of Asplund et al. (2009), not so much as needed to achieve a good fit when using an MHD case. For each particular case (HD or MHD) one should test if one could find a comparable level of consistency in the set of derived $\mathrm{C}$ and $\mathrm{O}$ solar abundance values as we have found here for the "200 G" MHD case.

The fair match of different observational quantities is an important result both in terms of confirming the quality of the MHD models we employ, as well as in relation to the conclusion presented in Paper III where its authors argued that it is still premature to consider a revision of solar abundances based on 3D MHD modelling. Interestingly, although our 3D magnetoconvection calculations employ the same code as used by them, our resulting photospheric models have a different temperature stratification than theirs. Differences in the number of opacity bins used, equation-of-state treatment, spatial resolution and temporal variation of the snapshots may contribute to those differences. Our models predict warmer temperatures in the middle photosphere, and as a consequence, a less steep temperature gradient in layers where many of the spectral lines that play a role in solar chemical abundance determination form. This can have a large effect on the emerging radiation spectrum, given that the excitation or ionisation processes involved in spectral line formation are predominantly dependent on that gradient. The results of this paper, namely the noticeable changes in the line spectra when going from HD to MHD and the excellent match that can be achieved in continuum and lines for the MHD models, together with further results in the literature like those of Papers I and II or those of Beck et al. (2013, 2015), which indicate an accurate average temperature stratification for our models with $\left\langle B_{z}\right\rangle$ between $\sim 50 \mathrm{G}$ and $\sim 100 \mathrm{G}$, confirm the need to consider magnetoconvection processes when solving problems that have a sensitive dependence on the shape of spectral features. 


\section{CONCLUSIONS}

In this work, we have studied the fit of various spectral features obtained through our 3D MHD numerical models of convection to observational constraints, finding, in general a very good agreement. This is particularly the case for absolute continuum intensity matching as well as for centre-to-limb variation in the wavelength range $600-2400 \mathrm{~nm}$. The adopted opacities and the 3D a posteriori radiation transfer processing prove to be important for a good match of observed solar continuum intensity from disc centre to limb. We have also shown that it is possible to achieve a good fit of the difficult [O I] $557 \mathrm{~nm}$ and $630 \mathrm{~nm}$ spectral features in spite of the blends and peculiarities of these lines. A convection model with the Asplund et al. (2009) abundances $\left[\log \epsilon(\mathrm{C})_{\odot}=8.43 \mathrm{dex}\right.$ and $\log \epsilon(\mathrm{O})_{\odot}=8.69$ dex] does not yield a satisfactory match, neither for the HD nor for any of the MHD cases. A better fit can be obtained by modifying the input abundances; for instance, a very good fit is obtained for our MHD "200 G" model using abundance values higher than those of Asplund et al. (2009) by several centidex. Also importantly, the presence of magnetic fields in the convection models is seen to cause non-negligible changes in the line profiles (especially in the [O I] 630-nm case). This effect occurs indirectly via the modification in the average temperature stratification of the atmosphere in the line-forming layers. The line asymmetries are also seen to be marginally different in the HD and MHD cases, so we cannot exclude the possibility that the differences in the average flow fields between the HD and MHD calculations play a role as well in modifying the line profile shapes. Adding to the results in this paper those from previous publications on abundances (Paper I; II), or those dealing with thermodynamic properties or polarization signals from our numerical models (Beck et al. 2013, 2015), we conclude that one should not neglect the magnetic field of the convection cells even in quiet Sun regions when aiming at accurate spectral modeling and chemical abundance determination.

We gratefully acknowledge financial support by the Spanish Ministries of Research and Innovation and of Economy through projects AYA2011-24808 and CSD2007-00050. We acknowledge the computing time granted through the DEISA SolarAct and PRACE SunFlare projects and the corresponding use of the HLRS (Stuttgart, Germany) and FZJ-JSC JUROPA (Jülich, Germany) supercomputer installations, as well as the computer resources and assistance provided at the MareNostrum (BSC/CNS/RES, Spain) and LaPalma (IAC/RES, Spain) supercomputers. We thank H. Uitenbroek for guidance with his $\mathrm{RH}$ code, and R. Rezaei, C. A. R. Beck and N. Vitas for useful discussions on the solar continuum intensity and related topics, as well as N. Shchukina and J. Trujillo Bueno for conversations about their recent work. We also acknowledge T. M. D. Pereira for clarifications on the 3D LTE line formation code and opacity package employed in Paper III. We have made use of the NIST ASD v5 available at http://physics.nist.gov/asd and of the first release (2014-02-05) of the "VALD3" data, as available from the web interface of the Vienna Atomic Line Database (VALD) project at http://vald.astro.univie.ac.at/ vald3/php/vald.php. Finally, we thank the anonymous referee for the helpful comments. 


\section{REFERENCES}

Asplund, M., Nordlund, Å., Trampedach, R., Allende Prieto, C., \& Stein, R. F. 2000, A\&A, 359, 729

Asplund, M. 2004, A\&A, 417, 769

Asplund, M. 2005, ARA\&A, 43, 481

Asplund, M., Grevesse, N., Sauval, A. J., Allende Prieto, C., \& Kiselman, D. 2004, A\&A, 417, 751

Asplund, M., Grevesse, N., Sauval, A. J., \& Scott, P. 2009, ARA\&A, 47, 481

Ayres, T. R. 2008, ApJ, 686, 731

Bastien, F. A. 2014, arXiv:1408.1112

Beck, C., Rezaei, R., \& Fabbian, D. 2011, A\&A, 535, A129

Beck, C., Fabbian, D., Moreno-Insertis, F., Puschmann, K. G., \& Rezaei, R. 2013, A\&A, 557, A109

Beck, C., Fabbian, D., Puschmann, K. G., \& Rezaei, R. 2015, A\&A, submitted

Beck, C., Fabbian, D., Puschmann, K. G., \& Rezaei, R. 2015, Sol. Phys., submitted

Bellot Rubio, L. R., \& Orozco Suárez, D. 2012, ApJ, 757, 19

Bruls, J. H. M. J. 1992, "Formation of diagnostic lines in the solar spectrum", Thesis Utrecht University, Utrecht, The Netherlands

Burlov-Vasilijev, K. A., Matvejev, Y. B., \& Vasiljeva, I. E. 1998, in Second Annual Lowell Observatory Fall Workshop, "Solar Analogs: Characteristics and Optimum Candidates", Hall, J. C. (Ed.), 115, available at

www2.lowell.edu/users/jch/workshop/kabv/kabv.html

Centeno, R., \& Socas-Navarro, H. 2008, ApJ, 682, L61

Criscuoli, S., Ermolli, I., Uitenbroek, H., \& Giorgi, F. 2013, ApJ, 763,144

Criscuoli, S. 2013, ApJ, 778, 27

Criscuoli, S., \& Uitenbroek, H. 2014, A\&A, 562, L1

Criscuoli, S., \& Uitenbroek, H. 2014b, ApJ, 788, 151

Dravins, D., Lindegren, L., \& Nordlund, A. 1981, A\&A, 96, 345

Dravins, D. 1982, ARA\&A, 20, 61

Dravins, D. http://arxiv.org/pdf/1408.1402v1.pdf

Fabbian, D., Asplund, M., Carlsson, M., \& Kiselman, D. 2006 A\&A, 458, 899

Fabbian, D., Asplund, M., Barklem, P. S., Carlsson, M., \& Kiselman, D. 2009, A\&A, 500, 1221

Fabbian, D., Khomenko, E., Moreno-Insertis, F., \& Nordlund, Å. 2010, ApJ, 724, 1536

Fabbian, D., Moreno-Insertis, F., Khomenko, E., \& Nordlund, Å. 2012, A\&A, 548, 35

Freytag, B., Steffen, M., \& Dorch, B. 2002, Astronomische Nachrichten, 323, 213

Gošić, M., Bellot Rubio, L. R., Orozco Suárez, D., Katsukawa, Y., \& Del Toro Iniesta, J. C. 2014, arXiv:1408.2369

Gray, D. F. 1992, Camb. Astrophys. Ser., Vol. 20,

Gurtovenko, E. A., \& Kostyk, R. I. 1989

Kitiashvili, I. N., Kosovichev, A. G., Mansour, N. N., \& Wray, A. A. 2013, arXiv:1312.0982

Koesterke, L., Allende Prieto, C., \& Lambert, D. L. 2008, ApJ, 680,764

Kramida, A., Ralchenko, Yu., Reader, J. and NIST ASD Team 2013. NIST Atomic Spectra Database (version 5.1), [Online]. Available: http://physics.nist.gov/asd

Kupka, F., Piskunov, N., Ryabchikova, T. A., Stempels, H. C., \& Weiss, W. W. 1999, A\&AS, 138, 119

Leenaarts, J., Pereira, T. M. D., Carlsson, M., Uitenbroek, H., \& De Pontieu, B. 2013, ApJ, 772, 89
Leenaarts, J., Pereira, T. M. D., Carlsson, M., Uitenbroek, H., \& De Pontieu, B. 2013, ApJ, 772, 90

Meléndez, J., \& Asplund, M. 2008, A\&A, 490, 817

Neckel, H., \& Labs, D. 1994, Sol. Phys., 153, 91

Nordlund, Å., Stein, R. F., \& Asplund, M. 2009, Living Reviews in Solar Physics, 6, 2

Pereira, T. M. D., Asplund, M., \& Kiselman, D. 2009, A\&A, 508, 1403

Pereira, T. M. D., Asplund, M., Collet, R., et al. 2013, A\&A, 554, A118

Pereira, T. M. D., Leenaarts, J., De Pontieu, B., Carlsson, M., \& Uitenbroek, H. 2013, ApJ, 778, 143

Pierce, A. K., \& Slaughter, C. D. 1977, Sol. Phys., 51, 25

Pierce, A. K., Slaughter, C. D., \& Weinberger, D. 1977, Sol. Phys., 52, 179

Piskunov, N. E., Kupka, F., Ryabchikova, T. A., Weiss, W. W., \& Jeffery, C. S. 1995, A\&AS, 112, 525

Rempel, M. 2014, ApJ, 789, 132

Ryabchikova, T. A., Piskunov, N. E., Stempels, H. C., Kupka, F., \& Weiss, W. W. 1999, Physica Scripta Volume T, 83, 162

Sánchez Almeida, J., \& Martínez González, M. 2011, Solar Polarization 6, 437, 451

Scott, P., Asplund, M., Grevesse, N., \& Sauval, A. J. 2009, ApJ, 691, L119

Scott, P., Asplund, M., Grevesse, N., Bergemann, M., \& Sauval, A. J. 2014, arXiv:1405.0287

Shchukina, N., Sukhorukov, A., \& Trujillo Bueno, J. 2012, ApJ, 755,176

Shchukina, N., \& Trujillo Bueno, J. 2011, ApJ, 731, L21

Shchukina, N., \& Trujillo Bueno, J. 2015, A\&A, submitted

Socas-Navarro, H. 2001, Advanced Solar Polarimetry - Theory, Observation, and Instrumentation, 236, 487

Socas-Navarro, H., \& Norton, A. A. 2007, ApJ, 660, L153

Socas-Navarro, H., de la Cruz Rodriguez, J., Asensio Ramos, A., Trujillo Bueno, J., \& Ruiz Cobo, B. 2014, arXiv:1408.6101

Socas-Navarro, H. 2014, arXiv:1409.6910

Solanki, S. K. 2009, Solar Polarization 5: In Honor of Jan Stenflo, 405, 135

Solanki, S. K., Krivova, N. A., \& Haigh, J. D. 2013, ARA\&A, 51, 311

Stein, R. F., \& Nordlund, A. 1998, ApJ, 499, 914

Stein, R. F. 2012, Living Reviews in Solar Physics, 9, 4

Takeda, Y., \& Ueno, S. 2012, Sol. Phys., 281, 551

Thaler, I., \& Spruit, H. C. 2014, A\&A, 566, A11

Thornton, L. M., \& Parnell, C. E. 2011, Sol. Phys., 269, 13

Trujillo Bueno, J., Shchukina, N., \& Asensio Ramos, A. 2004, Nature, 430, 326

Trujillo Bueno, J., \& Shchukina, N. 2009, ApJ, 694, 1364

Uitenbroek, H. 2001, ApJ, 557, 389

Uitenbroek, H. 2002, ApJ, 565, 1312

Uitenbroek, H., \& Criscuoli, S. 2013, Mem. Soc. Astron. Italiana, 84,369

Vögler, A., Shelyag, S., Schüssler, M., et al. 2005, A\&A, 429, 335

Vögler, A., Schüssler, M., 2007, A\&A, 465, L43

Wilhelm, K., \& Dwivedi, B. N. 2014, New A, 31, 8

Yelles Chaouche, L., Moreno-Insertis, F., \& Bonet, J. A. 2014, A\&A, 563, A93 